Apidologie, 1976, 7 (2), 139-149.

\title{
VERGLEICHENDE UNTERSUCHUNGEN ZUR TEMPERATURPRAEFERENZ VON INGEZÜCHTETEN UND NICHT-INGEZÜCHTETEN ARBEITERINNEN DER HONIGBIENE (APIS MELLIFICA)
}

\section{Comparaison du thermopreferendum chez des ouvrières d'abeille} (Apis mellifica) consanguines et non consanguines

\section{Dorothea BRÜCKNER}

Institut für Bienenkunde 637 Oberursel/Ts., Im Rothkopf 5 und Zoologisches Institut der Universität 8 München 2, Seidlstr. 25

\section{SUMMARY}

COMPARATIVE STUDY OF THE THERMOPRAEFERENDUM OF INBRED AND NON-INBRED WORKERS OF THE HONEYBEE (Apis mellifica L.)

The thermopraeferenda of inbred and non-inbred workers of Apis mellifica carnica were compared to find out if the inferior temperatureregulation of inbred workers found in former experiments is due to a change in "Sollwert" of these bees. The inbred workers had an inbreeding coefficient (F) of $87,5 \%$ : the queens of these bees had been inbred by instrumental insemination over several generations.

Workers of different ages $(6,7,8,13,14,15$ days old) were subjected to a temperature gradient in which they could select their thermopraeferendum Either single bees or groups of 2 inbred and 2 non-inbred bees were tested. No differences in thermopreferendum between inbred and non-inbred workers were found in these experiments. Since the thermopraeferendum is equal to the "Sollwert " it can be concluded from these experiments that there is no change in " Sollwert " of the inbred workers. In experiments in which the thermoregulation of groups of workers from the same colonies was measured the non-inbred workers reached significantly higher temperatures than the inbred workers. It is highly likely, that this is due to a deficiency in the production of metabolic heat of the inbred workers. 


\section{ZUSAMMENF ASSUNG}

Um die Frage zu klären, ob die in vorangegangenen Untersuchungen gefundene schlechte Temperaturregulierung von Inzuchtarbeiterinnen der Honigbiene (Apis mellifica) durch eine Verschiebung des Sollwertes bei diesen Arbeiterinnen bedingt ist, wurden die Temperaturpraeferenzen von ingezüchteten und nicht-ingezüchteten Arbeiterinnen vergleichend ontersucht. Die ingezüchteten Arbeiterinnen hatten einen Inzuchtkoeffizienten von $87,5 \%$, da die Königinnen ihrer Völker über mehrere Generationen durch künstliche Besamung ingezüchtet worden waren. Arbeiterinnen verschiedenen Alters $(6,7,8,13,14,15$ Tage alt) konnten in einem Temperaturgradienten (Temperaturorgel) ihre Praeferenztemperatur aufsuchen. Es wurden entweder einzelne Bienen oder Gruppen von je 2 ingezüchteten und 2 nicht-ingezüchteten Arbeiterinnen in den Gradienten eingesetzt. Das Temperaturpraeferendum der Arbeiterinnen entspricht ihren Sollwerten, es ändert sich mit den Bedingungen im Stock und dem Alter der Bienen.

Bei keiner der untersuchten Altersgruppen wurden Unterichiede in den Temperaturpraeferenzen von Inzucht- und Nicht-Inzuchtarbeiterinnen gefunden. Bei Messungen an Gruppen von je 50 Arbeiterinnen derselben Völker stellte sich jedoch heraus, daß die NichtInzuchtarbeiterinnen signifikant höhere Temperaturen erreichten als die Inzuchtarbeiterinnen. Aus diesen Ergebnissen muß geschlossen werden, daß bei Inzuchtarbeiterinnen keine Verschiebung der Sollwerte vorliegt, sondern daß die schlechtere Temperaturregulierung andere Ursachen hat. Wahrscheinlich ist die Abgabe an Stoffwechselwärme bei Inzuchtarbeiterinnen verringert.

\section{EINLEITUNG}

Das Temperaturregulierungsvermögen der Honigbiene ist als genau arbeitendes System bekannt, die Brutnesttemperatur wird auch bei großen Schwankungen in der Umgebungstemperatur bei ca $35{ }^{\circ} \mathrm{C}$ gehalten (Lit. bei Wilsov, 1971). Untersuchungen zur Temperaturregulierung der Arbeiterinnen in Abhängigkeit von ihrer genetischen Variabilität zeigten aber, daß Arbeiterinnen aus stark ingezüchteten Völkern die Temperatur weniger gut regulieren als solche aus nicht-ingezüchteten Völkern (BR ÜCKNER, 1975a). Bei niedrigen Umgebungstemperaturen ist die Temperatur des Brutnestes bei Inzuchtvölkern weniger hoch und schwankt stärker als bei Nicht-Inzuchtvölkern. Auch bei Messungen an kleinen Gruppen von Arbeiterinnen stellte sich heraus, daß die ingezüchteten Arbeiterinnen weniger hohe Temperaturen erreichten als die nicht-ingezüchteten Arbeiterinnen. Die schlechte Temperaturregulierung der ingezüchteten Arbeiterinnen muß durch ihre verringerte genetische Variabilität bedingt sein. Das Homozygotwerden zahlreicher Loci im Genom führt zu einem Mangel an Pufferungskapazität bei den Individuen (Lerner, 1954). Bei der Honigbiene konnte mangelnde Pufferung für ein morphologisches Merkmal i. e. die Rechts-linkssymmetrie der Flügel nachgewiesen werden : Bei stark ingezüchteten Arbeiterinnen treten bei diesem Merkmal, das ein Maß der intraindividuellen Variabilität ist, signifikant 
größere Schwankungen auf, als bei nicht-ingezüchteten Arbeiterinnen (BrüCKNer, 1975b). Die größere Asymmetrie der Flügel ingezüchteter Arbeiterinnen kommt dadurch zustande, daß die Individuen weniger gut gepuffert auf Störungen, die während der Entwicklung auftreten, zu reagieren vermögen. Auch das Merkmal Temperaturregulierung ist abhängig von einer Pufferungskapazität der Bienen. Einerseits können Störungen während der Entwicklung dabei eine Rolle spielen, andererseits sind stoffwechselphysiologische Vorgänge im adulten Tier von größter Bedeutung. Der Mangel an Regulierfähigkeit kann durch einen Mangel an Heizleistung i. e. Abgabe von Stoffwechselwärme bedingt sein. Für letztere Annahme spricht die Tatsache, daß bei den ingezüchteten Arbeiterinnen ein stärkerer Abfall der erreichten Temperaturen mit der Zeit festgestellt wurde als bei den nicht-ingezüchteten Arbeiterinnen (Brückner, 1975a). Zudem wäre es denkbar, daß der Sollwert, also die Temperatur, die zum Beispiel im Brutnest einreguliert werden soll, bei ingezüchteten Arbeiterinnen niedriger liegt als bei nicht-ingezüchteten Arbeiterinnen. Um herauszufinden, ob der Sollwert der ingezüchteten Arbeiterinnen verschoben ist, wurde in der vorliegenden Arbeit die Temperaturpraeferenz von ingezüchteten und nicht-ingezüchteten Arbeiterinnen vergleichend untersucht. In einem Temperaturgradienten stellen sich die Arbeiterinnen auf eine bestimmte Temperatur ein. Dieses Temperaturpraeferendum ist jedoch kein konstanier Wert, sondern ändert sich mit dem Alter der Bienen und in Abhängigkeit von den Bedingungen im Stock (Heran, 1952, Versuche mit Apis $m$. carnica) : Bis zum Alter von 7 Tagen liegt die Temperaturpraeferenz der Arbeiterinnen, unabhängig davon, ob Brut vorhanden ist oder nicht, in Höhe der Brutnesttemperatur. Bei Arbeiterinnen im Alter von 7-20 Tagen liegt sie, sofern Brut im Volk vorhanden ist, weiterhin in Höhe der Brutnesttemperatur, ist jedoch keine Brut vorhanden, so schwankt sie zwischen $33^{\circ}$ und $35^{\circ} \mathrm{C}$. Nach dem 20. Lebenstag, wenn die Bienen Flugbienen geworden sind, sinkt die Temperaturpraeferenz auf Werte zwischen $20^{\circ}$ und $33^{\circ} \mathrm{C}$ ab. Allerdings sucht nur die einzelne Biene, die allein keine Möglichkeit zur Temperaturregulierung hat, im Temparaturgradienten ihr Praeferendum auf; die Gruppe von Arbeiterinnen verhält sich wesentlich anders : Setzt man 30 oder mehr Arbeiterinnen einem Temperaturgradienten aus, so bilden sie an einer beliebigen Stelle, unabhängig von der dort herrschenden Temperatur, eine Traube, in deren Zentrum bei Versuchen mit jungen Bienen unbekannten Alters eine Temperatur von $33^{\circ} \mathrm{C}$ einreguliert wurde (LAvie und Roth, 1953). Bei Versuchen zur Temperaturpraeferenz können daher nur einzelne Bienen oder sehr kleine Gruppen getestet werden.

Die Temperaturpraeferenz der Arbeiterinnen muß der Temperatur entsprechen, die sie zusammen mit anderen Arbeiterinnen im Stock einreguliert halten würde. Anders ist die Tatsache nicht zu erklären, daß Bienen in Abhängigkeit von dem Vorhandensein von Brut im Volk ihre Temperaturpraeferenz 
ändern : ist Brut vorhanden, entspricht die Praeferenz der Brutnesttemperatur. Man muß annehmen, daß die Brut als Auslöser auf die Arbeiterinnen wirkt und sie den Sollwert der Brutnesttemperatur genau einhalten läßt. Unter brutlosen Bedingungen wirkt die Gruppe allein als Auslöser für die Temperaturregulierung, die Arbeiterinnen halten eine niedrigere Temperatur als im Brutnest ein, wobei die Wintertraube eine spezielle Situation davon darstellt. Auch bei der von der Gruppe ausgelösten Temperaturregulierung müssen bestimmte Sollwerte zugrunde liegen, die Schwankungen um diese Sollwerte sind jedoch wesentlich größer als im Fall der Brutnesttemperatur.

Es ist sehr wahrscheinlich, daß die verschiedenen Sollwerte in den Individuen nebeneinander vorhanden sind, so daß die Temperaturregulierung je nach den Bedürfnissen des Volkes erfolgen kann.

Würden die Inzuchtarbeiterinnen nun andere Sollwerte besitzen als die Nicht-Inzuchtarbeiterinnen, so müßten sie sich im Temperaturgradienten auch auf andere Temperaturen einstellen als die Nicht-Inzuchtarbeiterinnen. Nach den Ergebnissen der Untersuchungen zur Temperaturregulierung müßte man erwarten, daß sie sich auf niedrigere Werte einstellen als die NichtInzuchtarbeiterinnen.

\section{MATERIAL UND METHODE}

Als Versuchsmaterial dienten Arbeiterinnen aus 2 ingezüchteten und 2 nicht-ingezüchteten Völkern von Apis mellifica carnica. Der Inzuchtkoeffizient der ingezüchteten Arbeiterinnen betrug $87,5 \%$. Zur Art der Kreuzungen, die nötig waren, um diesen Inzuchtkoeffizienten zu erreichen, siehe Brückner 1975a. Die Kreuzungen wurden alle mit Hilfe der künstlichen Besamung durchgeführt. Waben mit verdeckelter Brut wurden aus den Völkern entnommen und im Brutschrank bei $35{ }^{\circ} \mathrm{C}$ zum Schlüpfen gebracht. Die Inzuchtarbeiterinnen wurden markiert und je 100 Arbeiterinnen von einem Volk in Plexiglaskäfige (der Maße $9 \times 6 \times 5 \mathrm{~cm}$ ) gesetzt. Die Bienen wurden mit frischem Pollen, Wasser und Honig versorgt und bis zum Versuchsbeginn bei $30^{\circ} \mathrm{C}$ im Dauerdunkel gehalten. Für die Messungen der Temperaturpraeferenz wurde eine Temperaturorgel benutzt (Henter, 1953; Heran, 1952). Den Boden eines Laufganges (Maße $70 \times 4 \times 5 \mathrm{~cm}$ ) bildet eine Aluminiumplatte, die auf der einen Seite mit Eiswasser gekühlt, auf der anderen mit einem Thermostaten erhitzt wird. Der Laufgang ist mit einer Plexiglasplatte und roter Folie abgedeckt. Der Temperaturgradient, der sich in dem Laufgang einstellt, wird mit 6 Thermometern gemessen, die im Abstand von je $10 \mathrm{~cm}$ in $1 \mathrm{~cm}$ Höhe über dem Boden angebracht sind. In anderen Temperaturorgeln wird die Temperatur der Bodenplatte gemessen, da Bienen jedoch mit den Tarsen keine Wärme wahrnehmen können (die Temperaturwahrnehmung geschieht vor allem mit den letzten 5 Antennengliedern), erscheint es sinnvoller, die Lufttemperatur knapp über dem Boden zu messen. Während der Versuche wurde der Gradient konstant gehalten, so daß an der ersten Meßstelle stets $13{ }^{\circ} \mathrm{C}$ an der 6 . stets $45,5^{\circ} \mathrm{C}$ gemessen wurde. Bei der Auswertung wird ein linearer Verlauf der Temperaturen zwischen den Meßstellen angenommem.

Für die Versuche wurden entweder einzelne Arbeiterinnen oder Gruppen von je 2 Inzuchtund 2 Nicht-Inzuchtarbeiterinnen in der Mitte des Laufganges eingesetzt. Nach 30 Minuten wurde zum erstenmal festgestellt, wo sich die Bienen befanden, 4 weitere Ablesungen wurden im Abstand von jeweils 5 Minuten durchgeführt. Nur ruhig sitzende Bienen wurden für die Messungen berücksichtigt. Die Bienen der Gruppen waren während der Versuche 6,7 und 8 Tage alt, die Einzelbienen 13, 14 und 15 Tage.

Da sich die Temperaturpraeferenzen der Arbeiterinnen zwischen dem 12. und 16. Tag nur um $0,4{ }^{\circ} \mathrm{C}$ verschieben (Herax, 1952, Versuche mit Bienen aus brutlosen Völkern), wurden die 13, 14 und 15 Tage alten Arbeiterinnen bei der Auswertung als einheitliche Gruppe betrachtet. 
Außerdem wurden Messungen zur Temperaturregulierung an Gruppen von je 50 Bienen eines Inzucht- und eines Nicht-Inzuchtvolkes bei $20^{\circ} \mathrm{C}$ in kleinen Käfigen durchgeführt. Bienen derselben Völker waren auf ihre Temperaturpraeferenz hin getestet worden. Zum Messen der von den Bienen erreichten Temperaturen wurde ein Meßgerät benutzt, das einen Stechfühler besaß, der durch die seitlichen Luftlöcher in die Käfige eingeführt werden konnte. (Genaueres zur Methode siehe Brǘckner, 1975a). Die Temperaturen wurden stündlich über 3 Tage von 8-18 h gemessen. Zu Beginn der Messungen waren die Bienen 9 Tage alt. Bei den Ergebnissen werden alle Werte, deren Irrtumswahrscheinlichkeit unter $5 \%$ liegt, als signifikant bezeichnet.

\section{ERGEBNISSE}

\section{Messungen mit je 4 Arbeiterinnen}

Der Vergleich der Mittelwerte der Temperaturpraeferenzen von Inzuchtund Nicht-Inzuchtarbeiterinnen zeigt, daß keine signifikanten Unterschiede bestehen. In 'Abb. 1, 2 und 3 sind die von 6, 7 und 8 Tage alten Arbeiterinnen im Gradienten gewählten Temperaturen aufgetragen. Der Gradient wurde für die Darstellungen in Temperaturstufen von $0,25{ }^{\circ} \mathrm{C}$ eingeteilt; dies ist für

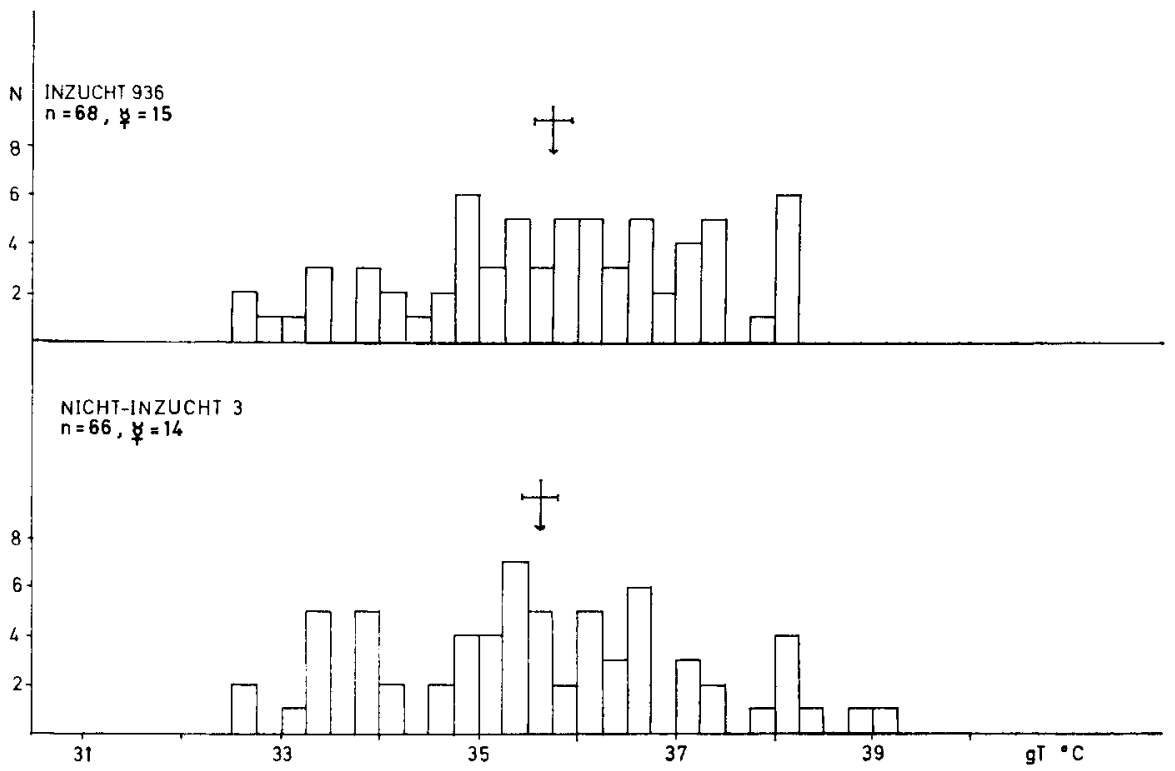

Aвв. 1. - Versuche mit je 2 Inzucht- und 2 Nicht-Inzuchtarbeiterinnen. Von 6 Tage alten Arbeiterinnen im Temperaturgradienten gewählte Temperaturen mit Angabe des Mittelwertes und dessen Streuung.

gT $=$ Gewählte Temperaturen, in Temperaturstufen von $0,25{ }^{\circ} \mathrm{C}$ eingeteilt.

N Anzahl der gewählten Temperaturstufen.

Frg. 1. - Expériences sur des groupes de 4 ouvrières : 2 consanguines et 2 non consanguines. Températures choisies dans le gradient de température par des ouvrières âgées de 6 jours; moyenne et dispersion des valeurs.

gT $=$ températures choisies. Les divisions sont de $0,25{ }^{\circ} \mathrm{C}$.

N $=$ Nombre de divisions choisies. 


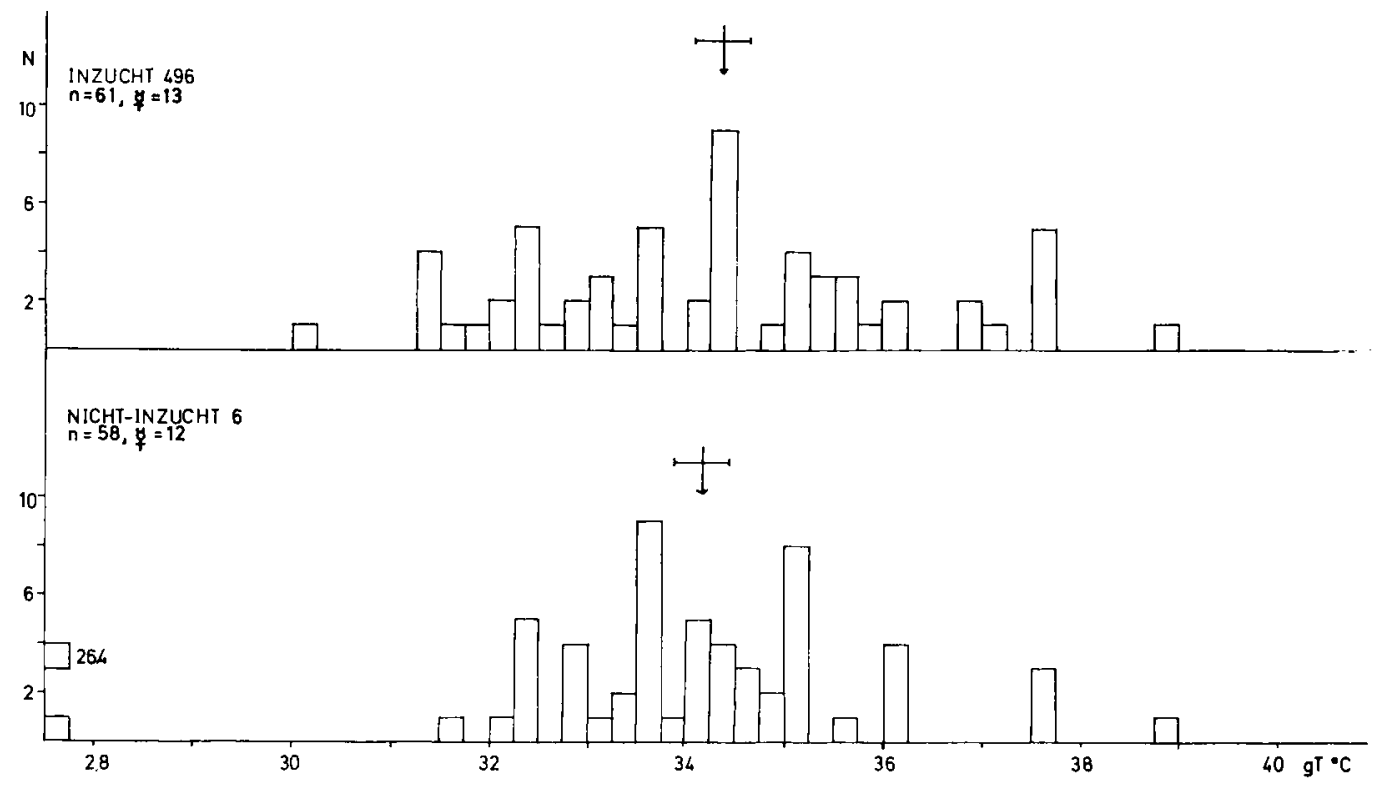

Aвв. 2. - Wie Abb. 1, Alter der Arbeiterinnen 7 Tage.

Fig. 2. - id. Fig. 1. Abeilles âgées de 7 jours.

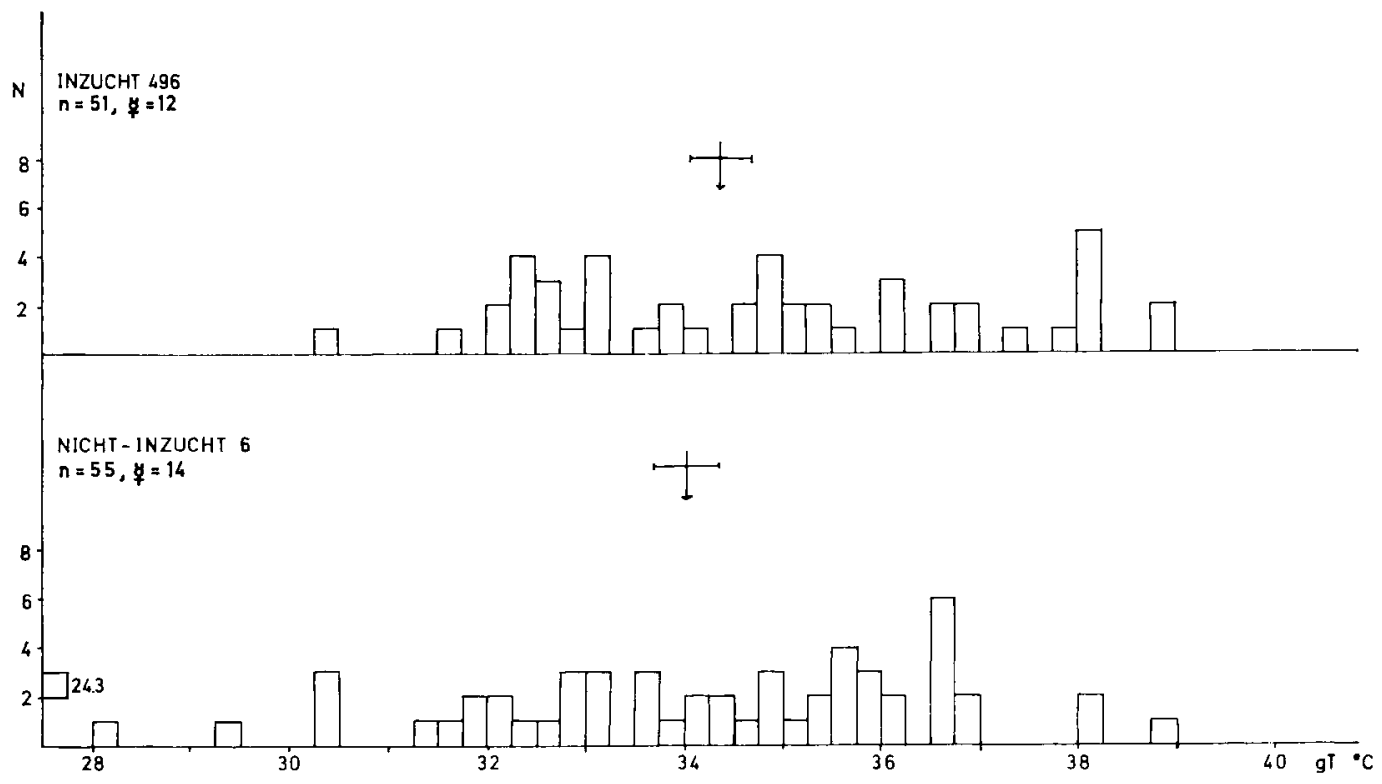

Авв. 3. - Wie Abb. 1, Alter der Arbeiterinnen 8 Tage.

Fig. 3. - id. Fig. 1. Abeilles âgées de 8 jours. 
die Arbeiterinnen die untere Grenze der Unterscheidungsmöglichkeit (HERAN, 1968). Die von Heran gefundene Tatsache, daß sich ab dem 7. Tag die Temperaturpraeferenz von Arbeiterinnen, die ohne Brut gehalten werden, ändert, indem die Werte absinken und stärker streuen, kann mit diesen Untersuchungen bestätigt werden. Vergleicht man die Mittelwerte der Temperaturpraeferenzen verschieden alter Arbeiterinnen, so stellt man fest, daß die Werte mit zunehmendem Alter der Bienen abnehmen, während die Varianzen der Werte zunehmen. Der Mittelwert der Temperaturpraeferenzen 6 Tage alter Bienen ist signifikant höher als der 7 und 8 Tage alter Bienen $(p<0,001)$, die Varianz ist signifikant kleiner $(p<0,001)$. Dies gilt sowohl für ingezüchtete als auch für nicht-ingezüchtete Arbeiterinnen.

Der Mittelwert der Temperaturpraeferenzen 6 Tage alter Arbeiterinnen (Inzucht und Nicht-Inzucht) entspricht den Mittelwerten der Brutnesttemperatur nicht-ingezüchteter Völker aus vorangegangenen Untersuchungen (BRÜCKNER, 1975a).

\section{Messungen mit einzelnen Arbeiterinnen}

In Abb. 4 sind die Mittelwerte der von jedem Individuum gewählten Temperaturen wiedergegeben. Auch bei diesen Messungen, bei denen jede

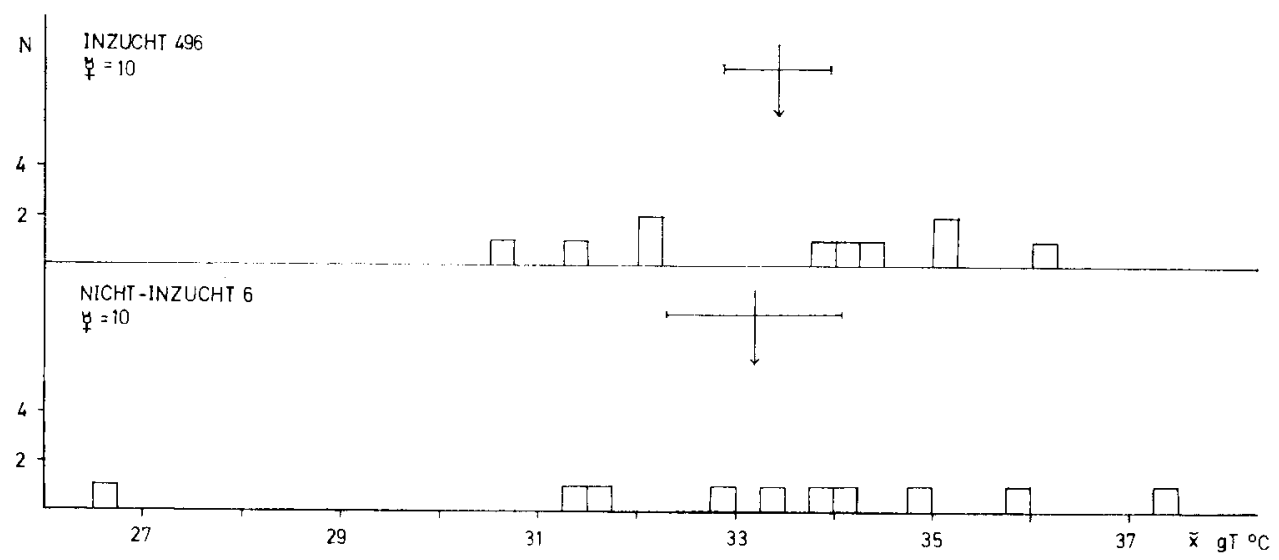

Aвв. 4. - Versuche mit Einzelbienen. Mittelwerte der im Temperaturgradienten gewählten Temperaturen für jedes Individuum mit Angabe des Mittelwertes der Mitelwerte und dessen Streuung. Alter der Arbeiterinnen 13 bis 15 Tage.

$\overline{\mathbf{x}} \mathrm{gT}=$ Mittel der gewählten Temperaturen für jedes Individuum, in Temperaturstufen von $0,25{ }^{\circ} \mathrm{C}$ eingeteilt.

N = Anzahl der im Mittel gewählten Temperaturstufen.

FIG. 4. - Expériences sur des abeilles isolées. Valeurs moyennes des températures choisies dans le gradient de température pour chaque individu. Moyenne et dispersion des moyennes. Abeilles âgées de 13 à 15 jours. $\begin{aligned} \overline{\mathbf{x}} \mathbf{g} \mathrm{T} & =\text { moyenne des températures choisies par chaque individu. Divisions de } 0,25^{\circ} \mathrm{C} \text {. } \\ \mathbf{N} & =\text { Nombre de divisions choisies en moyenne. }\end{aligned}$ 
mögliche Beeinflussung, die mehrere Bienen aufeinander haben könnten, ausgeschlossen ist, treten keine signifikanten Unterschiede in den Temperaturpaeferenzen von Inzucht- und Nicht-Inzuchtarbeiterinnen auf.

Im Gegensatz zu Heran, jedoch im Einklang mit anderen Autoren (LAvie und Roth, 1953) wurden bei Bienen aller getesteten Altersstufen relativ große Streuungen in den gewählten Temperaturen festgestellt. Die intraindividuelle und die interindividuelle Variabilität entsprechen sich dabei. Als Beispiel : Die interindividuelle Variabilität der Inzuchtarbeiterinnen der Einzelversuche ist aicht signifikant von ihrer intraindividuellen Variabilität verschieden. Einige Arbeiterinnen (z. B. eine Nicht-Inzuchtarbeiterin der Einzelversuche, Abb. 4) wählten auffallend niedrige Temperaturen. Dies kann auf folgende Tatsache zurückzuführen sein : Nach dem Einsetzen dringen manche Bienen, aufgeregt durch das Umsetzen in die Temperaturorgel, in den zu heißen Teil des Laufganges vor, aus dem sie zurückschrecken. Die Bienen setzen sich dann vielleicht unter dem Eindruck des Hitzeschocks, nicht aber unter dem Eindruck des Temperaturgradienten irgendwo zur Ruhe nieder (Heran, 1952).

\section{Messungen zur Temperaturregulierung}

Bei den dreitägigen Messungen zur Temperaturregulierung von je 50 Arbeiterinnen eines der Inzucht- und eines der Nicht-Inzuchtvölker zeigte sich, daß die Gruppe der Nicht-Inzuchtarbeiterinnen im Mittel signifikant höhere Temperaturen einreguliert hielt als die der Inzuchtarbeiterinnen ( $p<0,01$; Abb. 5). Diese Ergebnisse stimmen mit Ergebnissen aus vorangegangenen Untersuchungen überein, in denen die Nicht-Inzuchtarbeiterinnen stets signifikant höhere Temperaturen erreichten als die Inzuchtarbeiterinnen (BrücKNeR, 1975a). Wie in den vorangegangenen Versuchen sind auch bei diesen Messungen die Schwankungen in den erreichten Temperaturen bei den beiden Gruppen nicht signifikant verschieden, jedoch relativ groß. Die starken Schwankungen müssen durch die Kleinheit der Gruppen bedingt sein : 50 Arbeiterinnen sind nicht in der Lage, ein konstantes Temperaturnivau einreguliert zu halten.

Arbeiterinnen derselben Völker hatten im Temperaturgradienten keine unterschiedlichen Temperaturpraeferenzen gezeigt.

\section{DISK USSION}

Aus den Versuchen mit 6 Tage alten Arbeiterinnen geht hervor, daß zwischen Inzucht- und Nicht-Inzuchtarbeiterinnen kein Unterschied im Sollwert der Brutnesttemperatur besteht. Da die Brutnesttemperatur ein extrem wichtiger Wert für ein Volk ist — von ihm hängt die erfolgreiche 


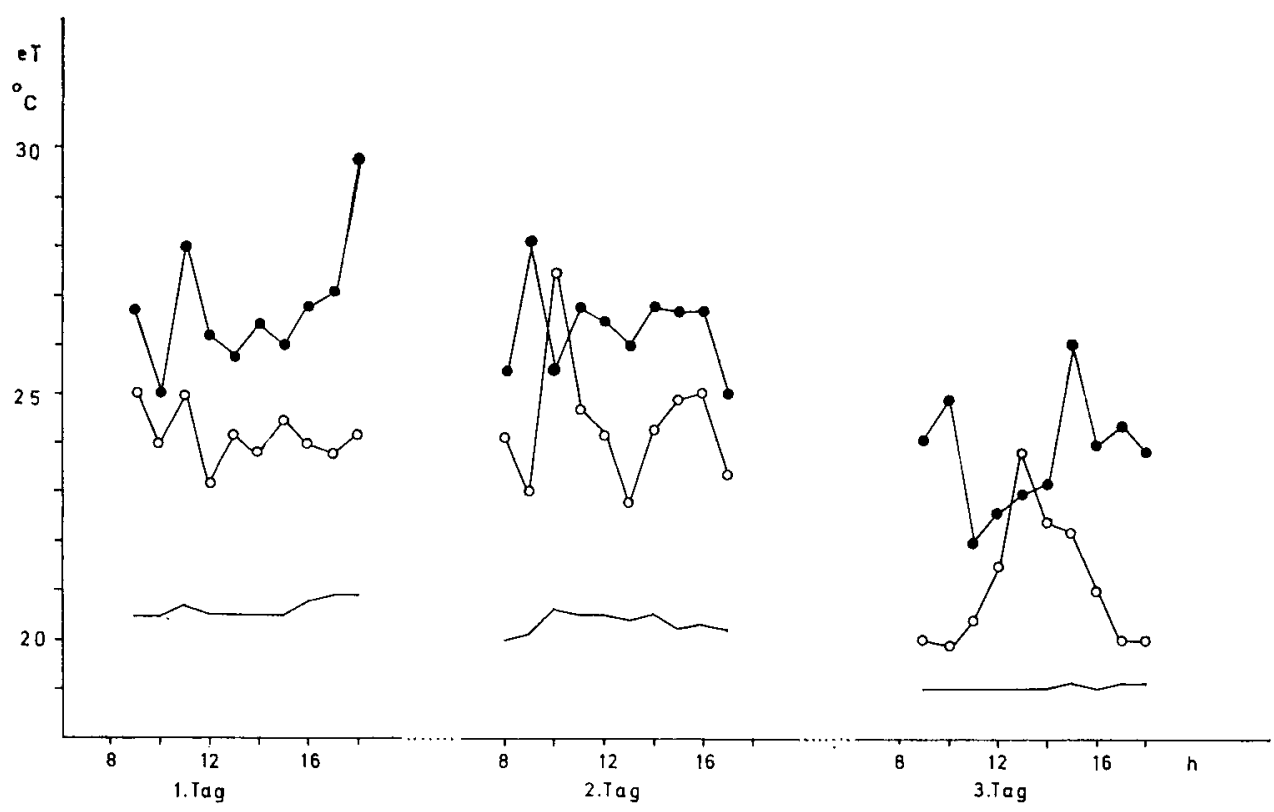

Aв8. 5. - Messungen zur Temperaturregulierung. Von je 50 Inzucht= und Nicht-Inzuchtarbeiterinnen erreichte Temperaturen (e $\mathrm{T}$ ).

Inzucht

Nicht-Inzucht

Umgebungstemperatur

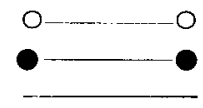

FIG. 5. - Mesures de la régulation thermique. Températures atteintes par des groupes de 50 ouvrières consanguines et des groupes de 50 ouvrières non consanguines.

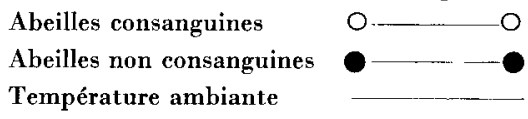

Aufzucht der Brut ab -, muß man annehmen, daß stets ein starker Selektionsdruck auf diesem Sollwert lag. Eine Verschiebung des Sollwertes der Brutnesttemperatur ist daher selbst bei Homozygotwerden zahlreicher Loci nicht unbedingt zu erwarten. Anders könnte es für die Sollwerte sein, die für Völker ohne Brut wichtig sind, sie liegen unter den Werten der Brutnesttemperatur und zeigen zudem wesentlich stärkere Schwankungen. Messungen an Bienen, die zwischen 7 und 15 Tage alt waren, haben jedoch gezeigt, daß sich auch die Temperaturpraeferenzen dieser Inzucht- und Nicht-Inzuchtarbeiterinnen nicht unterscheiden. Andererseits ergaben Messungen zur Temperaturregulierung von Gruppen von Arbeiterinnen derselben Altersklasse signifikante Unterschiede zwischen Inzucht- und Nicht-Inzuchtbienen. Aus diesen Ergebnissen muß man schließen, daß die Unterschiede in der Temperaturregulierung der Arbeiterinnen nicht auf einer Verschiebung der Sollwerte bei den Inzuchtarbeiterinnen beruhen, sondern eine andere Ursache haben müssen. 
Wie schon erwähnt, haben vorangegangene Untersuchungen gezeigt, daß die Pufferungskapazität der Inzuchta:beiterinnen gegenüber Störungen geringer ist als die der Nicht-Inzuchtarbeiterinnen und daß der Abfall der erreichten Temperaturen mit der Zeit bei Gruppen von Inzuchtarbeiterinnen größer ist als bei Gruppen von Nicht-Inzuchtarbeiterinnen. Da die Traubenbildung bei diesen Versuchen keine wesentliche Rolle spielte (BR̈̈CKNER, 1975a) sprechen die Ergebnisse dafür, daß die Abgabe an Stoffwechselwärme bei den Inzuchtarbeiterinnen geringer ist als bei den Nicht-Inzuchtarbeiterinnen. Das heißt, daß die Inzuchtarbeiterinnen zwar dieselbe Temperatur erreichen wollen wie die Nicht-Inzuchtarbeiterinnen, dazu aber physiologisch nicht in der Lage sind. Inwieweit auch eine Veränderung in der Fähigkeit, eine Traube zu bilden, bei den Inzuchtarbeiterinnen eine Rolle spielt, wurde nicht getestet. Es wäre jedoch denkbar, daß auch diese Reaktion, die der Erhaltung von Wärme in einem engen Bereich dient (Brutnest, Wintertraube) bei der Inzucht weniger effektiv ist als bei der Nicht-Inzucht.

\author{
Eingegangen im Januar 1976. \\ Reģu pour publication en janvier 1976.
}

\title{
DANK
}

Herrn Professor Dr. F. Ruttner möchte ich sehr herzlich für die Ermöglichung eines Arbeitsaufenthaltes im Institut für Bienenkunde in Oberursel danken, sowie Herrn Dr. V. MAuL für die Ứberlassung der ingezüchteten Bienenvölker.

\section{RÉSUMÉ}

A fin d'établir si la mauvaise régulation thermique, que l'on avait trouvée chez des ouvrières consanguines lors d'expériences antérieures, est causée par un déplacement de la norme de température, on a mené diverses expériences de comparaison portant sur le thermopreferendum des ouvrières de 2 colonies consanguines et de 2 colonies non consanguines d'Apis mellifica carnica. Les ouvrières consanguines avaient un coefficient de consanguinité (F) de $87,5 \%$, puisqu'elles provenaient de colonies dont les reiness avaient été rendues consanguines sur plusieurs générations par insémination artificielle.

Les ouvrières ont été élevées de la façon suivante : on a mis des rayons de couvain operculé à éclore à $35^{\circ} \mathrm{C}$ et à leur éclosion les ouvrières ont été placées par groupes de 100 dans des cages en plexiglass. On les a approvisionnées en pollen frais, en eau et en miel et maintenues à $30^{\circ} \mathrm{C}$ dans l'obscurité jusqu'au début de l'expérience. Pour les expériences, qui ont été faites sur des ouvrières âgées de $6,7,8,13,14$ et 15 jours, les abeilles ont été placées dans un gradient de température soit isolément, soit par groupes de $4: 2$ ouvrières consanguines et 2 non consanguines. Dans le gradient de température les abeilles se trouvaient à l'emplacement de leur thermopreferendum. Mais celvi-ci n'est pas constant; il varie en fonction des conditions qui règnent à l'intérieur de la ruche et de l'âge des abeilles. Jusqu'à 7 jours il correspond à la température du nid à couvain, entre 7 et 20 jours il se situe entre 33 et $35^{\circ} \mathrm{C}$ dans la mesure où il n'y a pas de couvain dans la ruche, et chez des abeilles plus âgées il se trouve entre $20^{\circ}$ et $33^{\circ} \mathrm{C}$. 
On a pu observer des différences entre les thermopreferendums des ouvrières consanguines et ceux des ouvrières non consanguines, aussi bien dans les expériences avec 4 abeilles que dans celles avec une abeille isolée. Le thermopreferendum des abeilles âgées de 6 jours, consanguines ou non, se situait aux alentours de la température du nid à couvain. Quand l'âge des abeilles croît, il diminue tandis que la dispersion augmente.

Dans les recherches sur la régulation thermique des groupes de 50 abeilles, il est apparu que les ouvrières non consanguines atteignaient des températures nettement plus élevées que les ouvrières consanguines; des ouvrières provenant des mêmes colonies n'avaient montré dans le gradient de température aucune différence concernant le thermopreferendum. On doit conclure de ces résultats que la régulation thermique insuffisante des ouvrières consanguines n'est pas due à un déplacement de la norme de température. Il est vraisemblable que les ouvrières consanguines dégagent moins de chaleur métabolique que les non consanguines. Les faits attestent qu'il existe une insuffisance de capacité d'amortissage chez les ouvrières consanguines, ainsi qu'une forte chute dans les températures atteintes proportionnellement au temps (BrǘckNer, 1975a). On n'a pas cherché à savoir, mai cela serait possible, si la formation de la grappe est moins effective chez les ouvrières consanguines que chez les non consanguines.

\section{LITERATURVERZEICHNIS}

BrüCKner D., 1975a. Die Abhängigkeit der Temperaturregulierung von der genetischen Variabilität der Honigbiene (Apis mellifica) Apidologie 6 (4), 361-380.

BRÜCKNER D., 1975b. The influence of genetic variability on wing symmetry in honeybees (Apis mellifica) Evolution (im Druck).

Heran H., 1952. Untersuchungen über den Temperatursinn der Honigbiene unter besonderer Berücksichtigung der Wahrnehmung strahlender Wärme. Z. vergl. Physiol., 34, 179-206.

Heran H., 1968. in Traité de Biologie de l'Abeille I, Herausgeb. Remy Chauvin, Masson, 185-199.

Herter K., 1953. Der Temperalursinn der Insekten. Berlin, Duncker und Humboldt.

LAvie P. und Roth M., 1953. Sur le thermopraeferendum et la production de chaleur chez les abeilles. Physiol. comp. et ocol., 3, 57-62.

Lerner I. M., 1954. Genetic Homeostasis. New York, John Wiley.

Wilson E. O., 1971. The Insect Societies, Belknap Press, Cambridge Mass. chapter 16. 